\title{
Double-incident Angle Method for the Improvement of Angle Scan Surface Plasmon Resonance Sensor
}

\author{
Wei Luo ${ }^{1,2, a,{ }^{*}}$, Zelin $\mathrm{HU}^{2, \mathrm{~b}}$, Jiarui Liu ${ }^{3, c}$, Fenglong Sun ${ }^{1,2, \mathrm{~d}}$, Hualong $\mathrm{Li}^{1,2, \mathrm{e}}$, Lei \\ Chen $^{2, f}$, Huiyi Gao ${ }^{1, g}$ and Miao $\mathrm{Li}^{2, \mathrm{~h}}$ \\ ${ }^{1}$ Institute of Intelligent Machines, Chinese Academy of Sciences, Hefei 230031, China \\ ${ }^{2}$ University of Science \& Technology of China, Hefei 230026, China \\ ${ }^{3}$ University of Chinese Academy of Sciences, Beijing 100049, China \\ a`zjlw1018@mail.ustc.edu.cn, ${ }^{\mathrm{b}} \mathrm{hz}$ lin@ustc.edu.cn, ${ }^{\mathrm{C}} \mathrm{jrliu} 2010 @ 126 . c o m,{ }^{\mathrm{d}} \mathrm{sfl} @$ mail.ustc.edu.cn, ${ }^{\mathrm{e}}$ lih

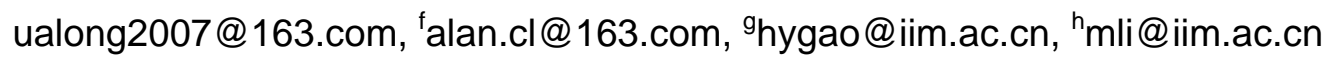

Keywords: Double-incident Angle Measurement Technique, Surface Plasmon Resonance, Refraction Index.

\section{Abstract.}

A double-incident angle measurement technique based on the Surface Plasmon Resonance (SPR) is described and experimentally demonstrated to measure the refraction index change of glucose versus the temperature in this paper. The angle scan SPR structure with parallel optical path is set up to carry out the experiment. Experiment reveals that the double-incident method has provided a new way to detect the small reflective index and may make the angle scan SPR real-timely monitor the molecular interaction possible.

\section{Introduction}

Since the Surface Plasmon Resonance (SPR) phenomenon is first demonstrated by Otto in 1968 [1] and made available for instrument application in 1990 by Biacore, the technique has been ideally applied in a wide range area, including the chemical and biology sciences, such as monitoring of adsorption process of molecules at metal surface, analysis of bio-molecular interaction and so on, for its advantages of label-free, real-time and high sensitivity [2-5]. In the case of the most often used Kretschmann configuration, a p-polarized light beam impinges onto the sensor (made from metal film coated on a glass slide) passing through a prism at a suitable incident angle (resonance angle), and undergoes attenuated total internal reflection (ATR) at a metal/prism interface. Then the evanescent wave penetrates the thin metal layer and resonates with the metal-dielectric surface plasmons propagating (SPP), causing the absorption of reflection light beam. The changes in the refractive index (RI) of the sensed medium will cause the coupling of evanescent wave different, leading the resonance angle shift. In this way, the SPR can prove an effective way to detect the change in RI. Glucose's RI has important applications [6], but the RI always changes tiny with temperature, making it too hard to be detected with the traditional SPR method [7]. Therefore, a higher accuracy method is required urgently.

In this paper, a double-incident angle technique is proposed for SPR sensor to improve the detection accuracy. The shape of typical resonance curve is similar with an asymmetric "V", which has two steep trends on both sides. And the most commonly used Kretschmann SPR is to measure the resonance angle shift. Besides, with two incident angles suitable chosen on each side of the "V", and the differential measurements at these two angles defined as the output of the system instead of resonance angle, a double-incident angle technique can be constructed [8]. Comparing with traditional measurements, the double-incident angle technique makes the experiment much more convenient, even without angle control mechanism. The technique also provides a higher sensitivity, leading especially suitable for detecting tiny RI changes. Furthermore, this technique may pave a new way for angle scan SPR system to monitor bio-molecular interaction on the sensor surface real-timely. 


\section{Theoretical analysis}

For a typical angular interrogating SPR system, a resonance curve indicating the reflectance intensity versus the incident light angle provides the resonance wave. The most often used SPR techniques are based on the measurement of the resonance angle's shift, which need to go through the whole incident angle to find the resonance angle. It would take too much time, and can't measure the RI change of medium in real-time. However, observing the resonance curve in Fig.1, when the incident angle increases, approaching the resonance angle, a sharp decline appears in the reflected light intensity, then, gradually increase away from the resonance angle. The rising speed is slower than the falling, forming an asymmetric ' $\mathrm{V}$ '. It is obvious that on each side of the resonance angle, the reflectance intensity changes faster than other places, which reveals that this area has a higher sensitivity. Therefore, a double-incident angle method can be constructed: two incident angles in the resonance angle's opposite sides are suitably chosen, where the reflected light intensity changes fastest, then the difference intensities of the reflected light is measured at this two different angles as the sensor output to instead of measuring the resonance angle shift.

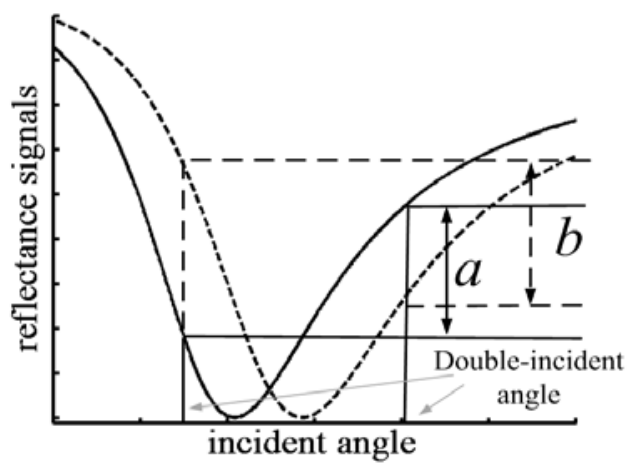

Fig.1. Outputs of the double-incident angle method: $a$ and $b$ are correspond to the two curves

Comparing with the traditional measurement of the resonance angle shift, the double-incident angle measurement takes full advantage of the data obtained at two incident angles and has a higher sensitivity. Noting that waveform distortion or resonance angle shifts may cause the two angles turn into the same side of the resonance angle, which would make the technique lose significance. In conclusion, the resonance angle should change in the range of the selected double-incident angles. By using the difference intensity as sensor outputs, the double-incident angle measurement has a great advantage in measuring the small reflective index change.

\section{Experiments}

The experiment setup, as shown in Fig. 2, is consisted of a fixed-wavelength laser, a polarizer, prism, a photo-detector array and the temperature controller module. The incident light generated by the He-Ne laser has a wavelength of $632.8 \mathrm{~nm}$, and the signal detector is a 2048 element linear-array charge-coupled device (TCD1208) with a USB interface as the data transport and power supplier making the system simplified. With a prism $(n=1.512)$ coated with $50 \mathrm{~nm}$ gold layer and a reflector making the in/out light parallel, the Kretschmann SPR structure is constructed.

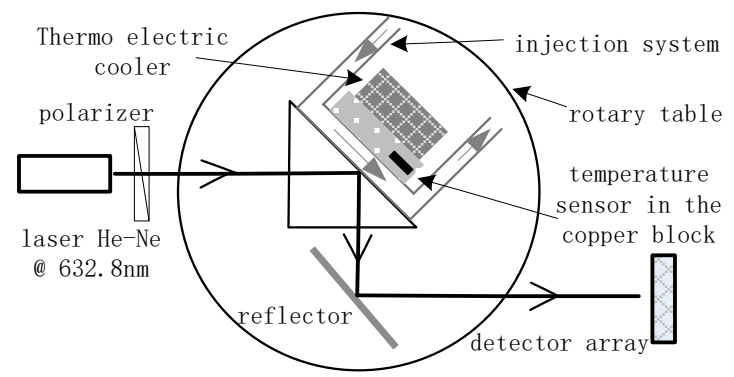

Fig.2. Surface Plasmon Resonance arrangement with parallel optical path 
To explore the temperature dependence of the RI variation of C6H12O6, the temperature must be carefully controlled. Stabilizing the temperature at a certain value needs a temperature controller and temperature sensor. Thermo electric Cooler (TEC) is a semiconductor with the Peltier effect. Both of the cooling and heating, as well as the rates of them, are determined by the direction and magnitude of the current. Therefore, after setting a TEC as the executor and the platinum resistance (APT1000) as the temperature sensor, a closed-loop temperature control system can be constructed. With the differential amplifier, the accuracy can reach 0.0001 degree. Thus, an organic system is formed.

\section{Result and discussion}

Double-incident angle method to detect the small reflection index change. The double-incident angle method is applied to measure the $2 \%$ (quality percentage) changes of glucose RI with different temperatures. The glucose is injected into the fluid cell, and the temperature is controlled at $20^{\circ} \mathrm{C}$. Then, the intensity of reflected light can be measured by traversing the entire incident angles with the CCD detector and the resonance curve (line a) is shown in Fig. 3. The same way is used to get the resonance curve (line b) at the temperature of $25{ }^{\circ} \mathrm{C}$ as shown in Fig. 3. Subtract the each reflectance signal in line ' $a$ ' from the corresponding value in line ' $b$ ', a difference cure is obtained as shown in Fig. 4. It is easy to observe that the maximum and minimum values of the difference cure, where the reflected intensity changes faster than others. Since the contour of the reflection index curve strongly depend on the prism, coupling method of gold film, and so on, once the system is ascertained, the variation of the curve will be certain. Therefore, the two incident angles corresponding to the maximum and minimum values of the difference cure can be applied to character the change of glucose's RI versus the temperature in the range of $20{ }^{\circ} \mathrm{C}-25{ }^{\circ} \mathrm{C}$, so, the RI changes with the temperatures can be detected by measuring the difference intensities at the two angles as the sensor outputs. The linear relation curves between the temperature and the double-incident signal is shown in Fig.5, it is obvious that the sensor output changes very linearly with temperature, and the fitting equation is $y=-17.9984 x+410.6337$, which is more sensitive than the relationship between the temperature and resonance angle shown in Fig 6.

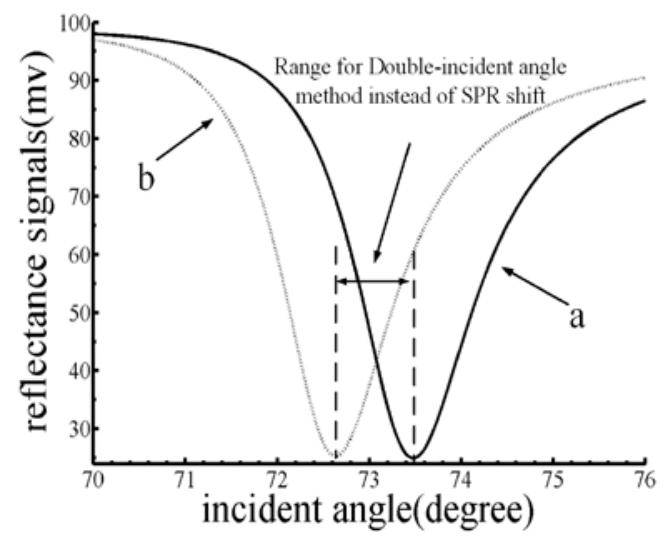

Fig.3. the resonance curve of $2 \%$ glucose at the temperature of $20^{\circ} \mathrm{C}$

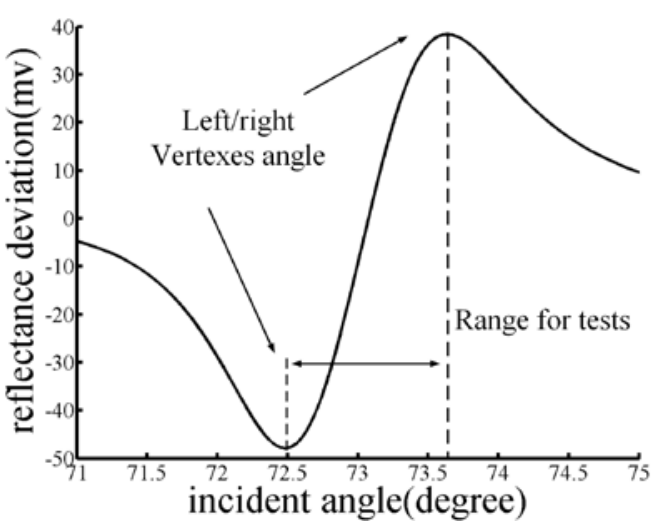

Fig.4.The differential of the resonance curves of the glucose at the temperature of $20^{\circ} \mathrm{Cand} 25^{\circ} \mathrm{C}$ 


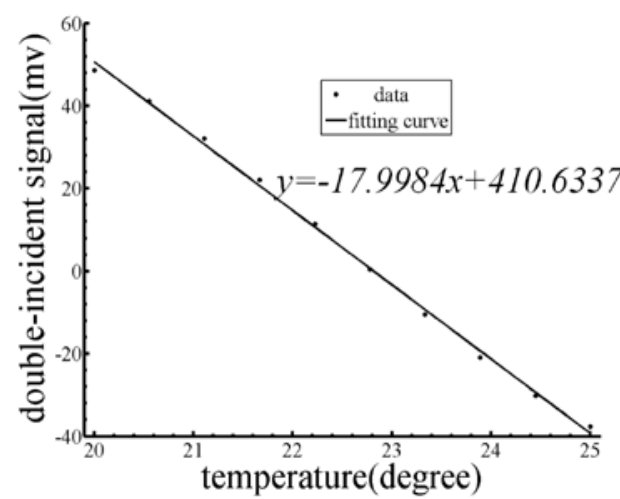

Fig.5. Linear fitting between the temperature and the signal of

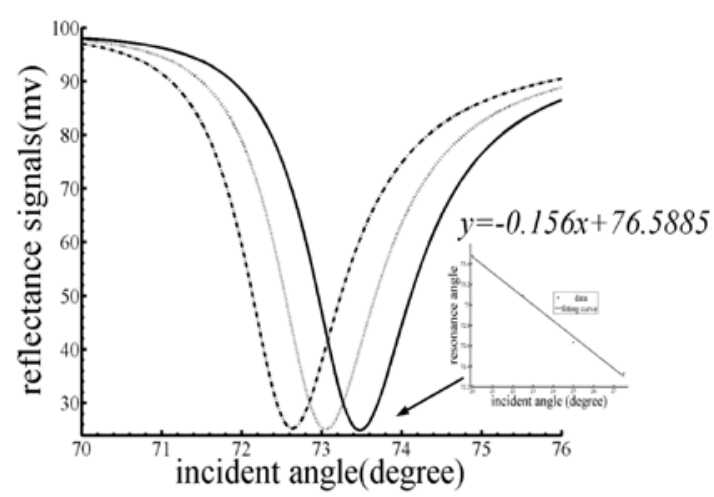

Fig.6. Linear fitting for the resonance angle shift with

In conclusion, by first determining the scope of extreme refractive index changes, two resonance curves can be obtained, and the double incident angles are found from the difference of them. Then, the different intensities at these two angles as the sensor outputs are measured to character the variation of the reflective index indirectly, instead of looking for resonance through the whole range of angle. In this way, it not only reduces the time but also provides a higher-sensitivity approach to detect tiny RI change.

Note to the Double-incident angle method. Noting that the double-incident angle may only be efficient in the situation that the resonance angle is in the range of the two incident angles. If out of the range, the output signal may not consistent with the actual situation, and the detail analyze is shown below.

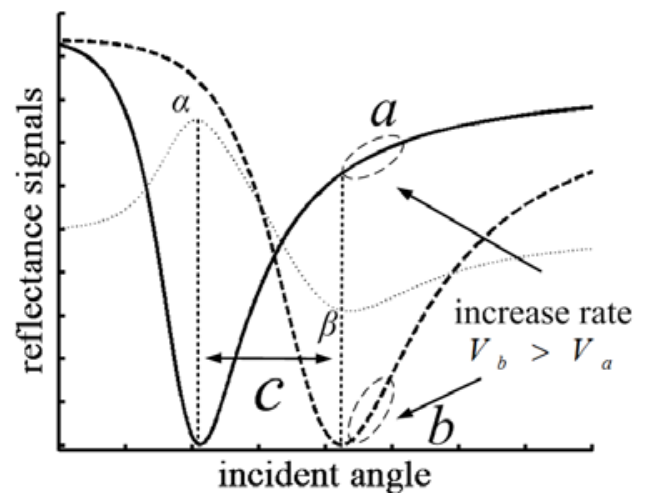

Fig.7. Schematic of the double-incident angle method's untrue output

From the curves in the Fig. 7, it is obvious that when the resonance angle comes greater than $\beta$ ( $\alpha$ and $\beta$ are the double-incident angles, $\alpha<\beta$ ), it will has a rapid variation that the increase velocity $v_{b}>v_{a}$, causing the double-incident angle decreases. Noting that the range of the double-incident angle shown in Fig. 7 is marked as the "C", beyond this range would lead the decline trend and the negative baseline.

\section{Summary}

A double-incident angle technique for SPR sensor is proposed and demonstrated experimentally, to be an effective method to measure the small changes of RI of glucose versus temperature. A linear relationship is found between the temperature and the double-incident sensor outputs, and the sensitivity is higher than the traditional method measuring the resonance angel shift. The particularly remarkable notice is also analyzed in detail. It would pave a way for the traditional angle scan SPR monitoring the RI change possible. 


\section{Acknowledgments}

The work is supported by the National High Technology Research and Development Program of China (863 Program, No. SS2013AA102302) and the Basic Research Program of Jiangsu Province (BK20131090).

\section{References}

[1] E. Kretschmann and H. Raether: Radiative decay of non radiative surface plasmon excited by light. Z. Naturforsch. Vol. 2135 (1968).

[2] X. Yang, Q. Wang, K. Wang, W. Tan, and H. Li: Enhanced surface plasmon resonance with the modified catalytic growth of Au nanoparticles. Biosens. Bioelectron. Vol. 1106-1110 (2007).

[3] Y. Liu and S. M. Chen: Compact Multi-channel Surface Plasmon Resonance Sensor for Real-time Multi-analyte Biosensing. Opt. Express. Vol. 20540-20548 (2015).

[4] C. Zhou and Y. Mu: A gravity-induced flow injection system for surface plasmon resonance biosensor. Talanta. Vol. 95-100 (2013).

[5] Marek Piliarik and Jiř́ Homola: Surface plasmon resonance (SPR) sensor: spproaching their limits? Opt. Express. Vol. 16505-16517 (2009).

[6] Z. Y. Song and G. Y. Feng: Accurate Measurement of the Refractive Index D-Glucose solution at Various Concentrations at Different Temperatures. Chinese Journal of Lasers. Vol. 1208008.1-1208008.5 (2014).

[7] F. Xiao and David Michel: Simultaneous Measurement of Refractive Index and Temperature Based on Surface Plasmon Resonance Sensors” Journal of Lightwave Technology. Vol. 3567-3571 (2014).

[8] X. Y. Zhang and K. Y. Wang: Double-incident angle technique for surface plasmon resonance measurements. Opt. Communications. Vol. 140-143 (2015).

[9] R. B. M. Schasfoort and A. J. Tudos: Handbook of surface plasmon resonance (Royal Society of Chemistry, 2008). 